\title{
COMUNICACIÓN
}

\section{Primeiro relato de Cryptosporidium spp. em emas (Rhea americana) cativas de zoológico no Brasil}

\author{
RODRIGO LUDWIG*, e SANDRA MÁRCIA TIETZ MARQUES** \\ FIRST REPORT OF Cryptosporidium spp. IN CAPTIVE RHEAS (Rhea americana) \\ IN A BRAZILIAN ZOO
}

The aim of this study was to evaluate the occurrence of Cryptosporidium spp. oocysts in common rhea (Rhea americana) living in captivity in the zoological park of the Zoobotanical Foundation of the State of Rio Grande do Sul, southern Brazil. Cryptosporidium spp. oocysts were detected in fecal smears of common rhea and in water samples by using the modified Ziehl-Neelsen staining method. The morphometric analysis of coccidia revealed small spherical oocysts, measuring, on average, $4.91 \mu \mathrm{m} \times 4.91 \mu \mathrm{m}$, and a length/width ratio of 1. The detection of Cryptosporidium spp. oocysts in fecal and water samples is important as it can indicate the transmission and maintenance of cryptosporidiosis in susceptible hosts. This is the first report of Cryptosporidium spp. in $\boldsymbol{R}$. americana in Brazil.

Key words: Cryptosporidium spp., oocysts, common rhea, zoo, Brazil.

\section{INTRODUÇÃO}

Emas (Rhea americana) são aves típicas da fauna brasileira e da América do Sul, habitam regiões de campos e savanas. Pertencem à Ordem Rheiforme, Família Rheidade e ao Gênero Rhea. No Brasil, as espécies mais freqüentes são a Rhea americana americana ${ }^{1}$, encontrada no norte do Paraná, regiões Nordeste, Centro-Oeste e Sudeste do Brasil e a Rhea americana intermedia, encontrada nos três estados da região $\mathrm{Sul}^{2}$. É uma ave pernalta de grande porte, considerada a maior ave brasileira, pertencente ao grupo das Ratitas - não voadoras. É um animal da fauna silvestre nativa, controlado e protegido por órgão governamental, que define e regulariza as normas para criação, além de proibir a caça.
Os parasitos são agentes patogênicos importantes, porém pouco se conhece sobre a sua distribuição nas populações silvestres. Protozoários do gênero Cryptosporidium são coccídeos zoonóticos obrigatórios que parasitam as células intestinais e do trato respiratório, são cosmopolitas e infectam cerca de 170 espécies animais. São reconhecidas, até o momento, 18 espécies e 40 genótipos $^{3}$. Os oocistos podem ser transmitidos entre hospedeiros susceptíveis através do contato fecal-oral ou ingestão de água e alimentos contaminados ${ }^{4}$.

Infecções por Cryptosporidium spp. já foram detectadas em mais de 30 espécies de aves de diferentes Ordens ${ }^{5,6}$. As espécies de Cryptosporidium relatadas são $C$. meleagridis, $C$. baileyi e $C$. galli sendo, recentemente, detectadas em

\footnotetext{
* Ciências Biológicas, Universidade do Vale do Rio dos Sinos (UNISINOS), São Leopoldo, Rio Grande do Sul, Brasil.

** Laboratório de Protozoologia, Departamento de Patologia Clínica Veterinária, Faculdade de Medicina Veterinárias da Universidade Federal do Rio Grande do Sul (UFRGS), Porto Alegre, Rio Grande do Sul, Brasil.
} 
pessoas com ou sem comprometimento do sistema imunológico ${ }^{7}$. Em Ratitas, o gênero Cryptosporidium não teve ainda o seu status estabelecido 8,9 .

Diversos zoológicos, parques de conservação e centros de reabilitação da vida selvagem têm demonstrado preocupação em relação à presença e o grau de contaminação de suas coleções de animais. $\mathrm{O}$ ambiente de zoológico, devido à concentração de diferentes espécies animais em espaços restritos, associado ao estresse do cativeiro e o contato direto com o homem, torna susceptível à infecção por Cryptosporidium spp. ${ }^{10}$. Exemplos são os relatos da criptosporidiose nos Zoológicos de Lisboa, Barcelona, Praga, Japão e Argentina ${ }^{10-13}$.

Por não ser exclusiva de países em desenvolvimento e por figurar no Programa de Doenças Negligenciadas da Organização Mundial da Saúde (OMS), a criptosporidiose torna-se uma protozoose com implicações legais para as autoridades de Vigilância Sanitária, devido ao seu impacto em Saúde Pública. Entretanto, o impacto sanitário e econômico desta zoonose ainda é indeterminado, principalmente porque alguns representantes do gênero Cryptosporidium não são espécie-específicos e facultam risco de transmissão para outros animais e para humanos ${ }^{9}$.

O Parque Zoológico da Fundação Zoobotânica do Rio Grande do Sul foi aberto ao público em 1962 e é uma das unidades de conservação mais visitadas do País; anualmente cerca de 600 mil pessoas visitam o local. São 160 hectares de área aberta à visitação pública. $\mathrm{O}$ acervo de répteis, aves e mamíferos é formado por 1.013 animais, incluindo espécies nativas e ameaçadas de extinção ${ }^{14}$. O Parque Zoológico é uma instituição que maneja e exibe coleções de animais silvestres vivos em ambientes que visam reconstituir as condições que cada espécie encontraria no ambiente natural, de acordo com a legislação proposta por órgão governamental, para a sobrevivência, reprodução e bem estar, com vistas à conservação, pesquisa, educação e lazer.

Este estudo objetivou investigar a ocorrência de oocistos de Cryptosporidium spp. em emas residentes no Parque Zoológico da Fundação Zoobotânica do Rio Grande do Sul e na água do lago do recinto onde vivem estes animais. O Parque localiza-se na região Metropolitana da Grande Porto Alegre, coordenadas 29 48'05.28"
S e $51^{\circ} 09^{\prime} 58.87^{\prime \prime} \mathrm{O}$, no município de Sapucaia do Sul, região Sul do Brasil.

\section{MATERIAL E MÉTODOS}

As amostras fecais pertenciam a emas adultas de ambos os gêneros, que aparentavam bom estado sanitário e nutricional no período do experimento. $\mathrm{O}$ recinto que abriga estes animais é muito amplo, área total de 8 ha, que se caracteriza pela presença de mata densa e por um lago que corre para outras áreas do Parque. Juntamente com as emas, habitam no local algumas capivaras, gansos, patos e uma anta.

Duas vezes por mês, entre março e maio de 2008 , foram coletados "pools" de amostras fecais de 23 emas, totalizando seis "pools" no período de três meses. Imediatamente após a defecação, fez-se o recolhimento das fezes do solo, o acondicionamento em frascos coletores e a marcação do animal com spray, evitando repetição da amostra em cada seção de colheita. As amostras fecais foram homogeneizadas no frasco coletor antes da execução dos esfregaços. A quantidade de indivíduos amostrados por «pool» dependeu do número de animais que defecaram no momento da colheita. Os seis "pools" variaram em número de indivíduos amostrados, de acordo com os valores listados a seguir: pool $01=13$; pool $02=18$; pool $03=15$; pool $04=16$; pool 05 $=14$; pool $06=12$. Realizaram-se esfregaços fecais em lâminas de vidro, os quais foram secos à temperatura ambiente; em seguida, fixados em álcool absoluto por cinco minutos. No laboratório os esfregaços fecais foram corados pela técnica de Ziehl-Neelsen modificada $(Z N m)^{15}$ e observados em microscópio óptico (modelo OLYMPUS CX40, Japan), em aumento de 1.000 $X$ (fator de correção de 3,6 ).

Do lago existente no recinto, foi coletada amostra de água da margem e acondicionada em frasco de vidro esterilizado, com capacidade de 1 litro. No laboratório, o volume total foi alicotado em tubos de ensaio de $10 \mathrm{ml}$, centrifugados por 10 minutos a 3.000 rotações por minuto em centrífuga (modelo ECONO-SPIN, marca DU PONT). Os sobrenadantes foram desprezados após centrifugação. Os sedimentos obtidos em cada tubo de ensaio foram colocados juntos em um novo tubo de $10 \mathrm{ml}$ e centrifugados até a redução do volume, em sucessivas centrifugações. O material decantado da última centrifugação foi 
pipetado em lâminas de vidro e colocado em estufa para a secagem. Posteriormente, as lâminas foram submetidas à técnica de coloração de ZNm, similar aos esfregaços fecais.

A análise morfométrica $(\mathrm{T})$ dos oocistos foi obtida através da mensuração com ocular micrométrica (modelo ERNST LEITZ, marca Wetzlar, Germany), acoplada ao microscópio óptico, levando-se em conta as medidas de comprimento (C) e largura (L), aplicando a fórmula $\mathrm{T}=\mathrm{C} / \mathrm{L}$ (ìm) ${ }^{16}$.

\section{RESULTADOS E DISCUSSÃO}

Através da confecção de esfregaços de fezes corados pela técnica de Ziehl-Neelsen modificada, pôde-se constatar a presença de oocistos de Cryptosporidium spp. (Figura 1). Do total de seis "pools", dois apresentaram oocistos. A presença de oocistos de Cryptosporidium spp. nestas amostras pode sugerir a manutenção da infecção, porque um mesmo animal infectado poderia ter colaborado com fezes nos dois grupos positivos. Viés inevitável, pela impossibilidade de isolar, em locais distintos, as emas que já haviam participado do experimento em colheitas anteriores.

A análise morfométrica revelou pequenos oocistos esféricos medindo, em média, $4,91 \mu \mathrm{m}$ X 4,91 $\mu \mathrm{m}$ e relação comprimento/largura de razão 1. Estes valores se assemelham aos descritos em avestruzes africanos importados pelo Canadá, com medida de 4,0-6,1 $\mu \mathrm{m}$ X 3,3$5,0 \mu \mathrm{m}^{8}$. Oocistos em fezes de aves infectadas na República Tcheca apresentaram médias de 6,2 X 4,6 $\mu \mathrm{m}$ para $C$. baylei, 5,2 X 4,6 $\mu \mathrm{m}$ para C. meleagridis e $8,3 \times 6,3 \mu \mathrm{m}$ para $C$. galli $^{17}$. Oocistos isolados de avestruzes no Brasil e submetidos à análise morfométrica mediram 6,0 X 4,8 $\mu \mathrm{m}(5,0-6,5$ X 4,2-5,3) com razão de $1,31^{18}$.

As lâminas obtidas da amostra de água foram positivas para oocistos de Cryptosporidium spp. O tamanho médio dos oocistos foi de 4,93 $\mu \mathrm{m} \mathrm{X}$ $5,25 \mu \mathrm{m}$ e relação comprimento/largura de razão 1,06 . Os oocistos medidos se encontram dentro dos limites do índice, de 1 a $1,4^{19}$.

Os coccídeos são, do ponto de vista econômico e sanitário, o mais importante grupo entre os protozoários. Em Ratitas, referências em relação à criptosporidiose ainda são escassas. O único relato de emas parasitadas por

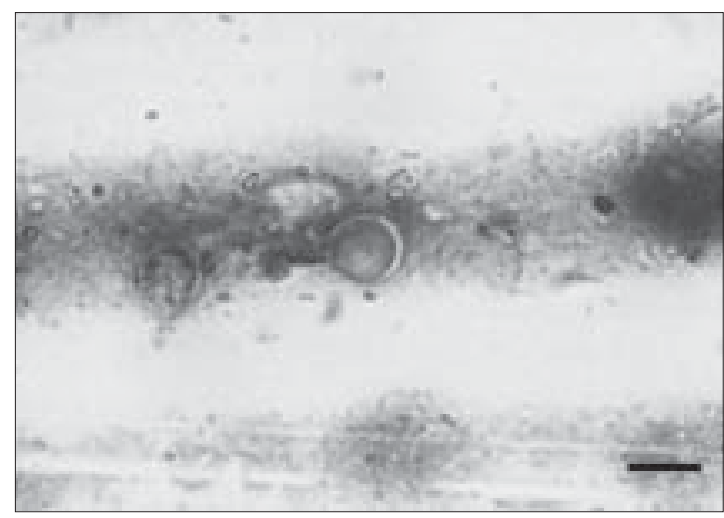

Figura 1. Oocisto de Cryptosporidium spp. corado pela técnica de Ziehl-Neelsen modificada (1000X; opt. 1,25). Escala: Bar $=10 \mu \mathrm{m}$. Fonte: Rodrigo Ludwig.

Cryptosporidium spp. ocorreu na Espanha, com $60 \%$ de prevalência em animais de criatórios comerciais ${ }^{9}$. Em avestruzes, aves que pertencem ao mesmo grupo das emas, a criptosporidiose também já foi registrada, porém as espécies ainda não foram determinadas ${ }^{20,21}$.

As condições de saúde, em determinadas populações, requerem melhorias em seu monitoramento, para avaliações precisas de seus impactos. A aplicabilidade da técnica de esfregaço fecal em serviços de saúde veterinária, saúde ambiental e controle epidemiológico, deveriam ser apreciados, principalmente, por se tratar de uma alternativa simples para detecção da criptosporidiose. Além de ser uma técnica diagnóstica de baixo custo e rápida de ser executada.

Existem outras metodologias de diagnóstico, mas devido ao alto custo tornam-se restritas a laboratórios com alta tecnologia, que priorizam trabalhos de pesquisa e identificação de novas espécies e genótipos. Embora, nestes casos, a utilização de esfregaços de fezes também seja uma ferramenta útil, porque pode discriminar animais positivos previamente, reduzindo custos e tempo na identificação de amostras positivas ${ }^{22,23}$.

A constatação da presença de oocistos de Cryptosporidium spp. é o primeiro passo para a detecção do foco zoonótico deste parasito ${ }^{24}$. No entanto, o grande desconhecimento da criptosporidiose como doença emergente e zoonótica, seus fatores epidemiológicos e a ausência de tratamento específico em todas as espécies hospedeiras ${ }^{25}$, dificulta o combate desta parasitose. 
Cryptosporidium spp. em emas (Rhea americana) cativas no Brasil - R. Ludwig e S. M. T. Marques

Apesar do pequeno tamanho amostral deste estudo, a presença de oocistos de Cryptosporidium spp. nas amostras fecais de emas ratifica que estes animais são hospedeiros e podem contribuir, juntamente com os oocistos encontrados na água, para a disseminação e contaminação ambiental. Além disso, o potencial zoonótico da criptosporidiose ganha maior importância porque estes animais mantêm contato com humanos em seu manejo e habitam um recinto formado por espécies mistas, dividindo o espaço com outros animais. O fato do lago deste recinto formar um curso d'água, que corre para outras áreas do Parque, torna-se um agravante na disseminação dos oocistos, por se manterem estáveis por longos períodos e serem resistentes aos mais variados métodos de tratamento de água. Desta forma, a via hídrica pode funcionar como uma facilitadora na disseminação dos oocistos, podendo atingir grande número de hospedeiros susceptíveis rapidamente ${ }^{26,27}$.

\section{RESUMO}

Este estudo objetivou avaliar a ocorrência de oocistos de Cryptosporidium spp. em emas (Rhea americana) cativas no Parque Zoológico da Fundação Zoobotânica do Rio Grande do Sul, sul do Brasil. Foram identificados oocistos de Cryptosporidium spp. em esfregaços de fezes de emas e em amostras de água, coradas pela técnica de Ziehl-Neelsen modificada. A análise morfométrica dos coccídeos revelou pequenos oocistos esféricos medindo, em média, 4,91 $\mu \mathrm{m}$ X 4,91 $\mu \mathrm{m}$ e relação comprimento/largura de razão 1. A confirmação da presença de oocistos nas amostras de fezes e de água é relevante pelo potencial de transmissão e manutenção da criptosporidiose em hospedeiros susceptíveis. Este é o primeiro relato de Cryptosporidium spp. em $R$. americana no Brasil.

\section{REFERÊNCIAS}

1.- DUNNING J S, BELTON W. Aves silvestres do Rio Grande do Sul. $3^{\circ}$ Ed. Porto Alegre, FZB/RS, 1993; 223.

2.- PARIZZI R C, SANTOS J M, OLIVEIRA M F, et al. Macroscopic and microscopic anatomy of the oviduct in the sexually mature Rhea (Rhea americana). Anat Histol Embryol 2008; 37: 169-76.

3.- XIAO L, RYAN U M. Molecular epidemiology. In: Fayer, R., Xiao, L. (Eds.), Cryptosporidium and
Cryptosporidiosis, second ed. CRC Press and IWA Publishing (Boca Raton, FL) 2008; 119-63.

4.- FAYER R, MORGAN U, UPTON S J. Epidemiology of Cryptosporidium: transmission, detection and identification. Int J Parasitol 2000; 30: 1305-22.

5.- O'DONOGHUE P J. Cryptosporidium and cryptosporidiosis in man and animals. Int J Parasitol 1995; 25: 139-95.

6.- FAYER R, SPEER C A, DUBEY J P. Avian cryptosporidiosis. In: Fayer, R. editor. Cryptosporidium and cryptosporidiosis. CRP Press (Boca Raton, FL), 1997; 1-33.

7.- DAL'OLIO A J, FRANCO R M B. Ocorrência de Cryptosporidium spp. em pequenos mamíferos silvestres de três áreas serranas do Sudeste brasileiro. Arq Bras Med Vet Zootec 2004; 56: 25-31.

8.- GAJADHAR AA. Cryptosporidium species in imported ostriches and consideration of possible implications for birds in Canada. Can Vet J 1993; 34: 115-6.

9.- PONCE GORDO F, HERRERA S, CASTRO A T, et al. Parasites from ostriches (Strutio camelus) and rheas (Rhea Americana) in Europe. Vet Parasitol 2002; 107: 137-60.

10.- APPELBEE A J, THOMPSON R C, OLSON M E. Giardia and Cryptosporidium in mammalian wildlifecurrent status and future needs. Trends Parasitol 2005; 21: $370-6$.

11.- ALVES M, XIAO L, LEMOS V, et al. Occurrence and molecular characterization of Cryptosporidium spp. in mammals and reptiles at the Lisbon Zoo. Parasitol Res 2005; 97: 108-12.

12.- MATSUBAYASHI M, TAKAMI K, KIMATA I, et al Survey of Cryptosporidium spp. and Giardia spp. infections in various animals at a zoo in Japan. J Zoo Wildl Med 2005; 36: 331-5.

13.- VENTURINI L, BACIGALUPE D, BASSO W, et al Cryptosporidium parvum em animales domésticos y en monos de um zoológico. Parasitol Latinoam 2006; 61: 90-3.

14.- FUNDAÇÃO ZOOBOTÂNICA DO RIO GRANDE DO SUL - FZB/RS. Disponível em: <http://www.fzb. rs.gov.br/zoologico/>. Acesso em: 07 ago. 2008.

15.- HENRICKSEN S A, POHLENZ J F L. Staining of Cryptosporidia by a modified Ziehl-Neelsen technique. Acta Vet Scand 1981; 22: 594-6.

16.- ELLIOT A, MORGAN U M, THOMPSON R C A. Improved staining method for detecting Cryptosporidium oocysts in stools using malechite Green. J General App Microbiol 1999; 45: 139-42.

17.- RYAN U M, XIAO L, READ C, et al. The redescription of Cryptosporidium galli Pavlasek, 1999 (Apicomplexa: Cryptosporidiidae) from birds. Aj. Parasitol 2003; 89: 809-13.

18.- SANTOS M M A B, PEIRÓ J R, MEIRELES M V. Cryptosporidium infection in ostriches (Struthio camelus) in Brazil: clinical, morphological and molecular studies. Brazilian J Poult Sci 2005; 7: 113-7.

19.- UPTON S J, CURRENT W L. The species of Cryptosporidium (Apicomplexa: Cryptosporiidae) infecting mammals. J Parasitol 1985; 71: 625-9.

20.- De GRAAF D C, VANOPDENBOSCH E, ORTEGA-MORA L M, et al. A review of the importance of criptosporidiosis in farm animals. 
Int J Parasitol 1999; 29: 1269-87.

21.- MORGAN U M, MONIS P T, XIAO L, et al. Molecular and phylogenetic characterization of Cryptosporidium from birds. Int J Parasitol 2001; 31: 289-96.

22.- COLE D J, COHEN N D, SNOWDEN K, SMITH R. Prevalence of and risk factors for fecal shedding of Cryptosporidium parvum oocysts in horses. J Amer Vet Med Ass 1998; 213: 1296-302.

23.- SUÁREZ-LUENGAS L, CLAVELA, QUÍLEZ J, et al. Molecular characterization of Cryptosporidium isolates from pigs in Zaragoza (northeastern Spain). Vet Parasitol 2007; 148: 231-5.

24.- GRACENEA M, GÓMEZ M S, TORRES J, et al. Transmission dynamics of Cryptosporidium in primates and herbivores at the Barcelona zoo: a long-term study. Vet Parasitol 2002; 104: 19-26.

25.- SAVIOLI L, SMITH H, THOMPSON A. Giardia and
Cryptosporidium join the «Neglected Diseases Initiative». Trends in paras 2006; 22: 203-8.

26.- FAYER R. Cryptosporidium: a water-borne zoonotic parasite. Vet Parasitol 2004; 126: 37-56.

27.- BORGES J C G, ALVES L C, FAUSTINO M A G, et al Ocorrência de oocistos de Cryptosporidium spp. na água destinada a manutenção dos peixes-boi (Trichechus manatus) em cativeiro. Biotemas 2007; 20: 67-74.

Agradecimentos: Aos funcionários do Parque Zoológico de Sapucaia do Sul pela colaboração durante a coleta das amostras. Ao responsável pelo Laboratório de Protozoologia da Faculdade de Medicina Veterinária da UFRGS, Prof. Dr. Flávio A. P de Araújo, por autorizar a utilização das dependências do mesmo. À laboratorista Vanessa Baptista do Laboratório de Histologia da UNISINOS, pela colaboração durante a realização das fotografias.

Correspondencia a:

Sandra Márcia Tietz Marques

E-mail: sandra.marques@ufrgs.br 